\title{
Stability of splay states for pulse-coupled neuronal networks: finite size versus finite pulse-width effects
}

\author{
Alessandro Torcini*1,3, Ruediger Zillmer ${ }^{1,3}$, Roberto Livi ${ }^{2,3}$ and \\ Antonio Politi ${ }^{1}$
}

\author{
Address: ${ }^{1}$ Istituto dei Sistemi Complessi - CNR, 50019 Sesto Fiorentino, Italy, ${ }^{2}$ Department of Physics, 50019 Sesto Fiorentino, Italy and ${ }^{3}$ Istituto \\ Nazionale di Fisica Nucleare, 50019 Sesto Fiorentino, Italy \\ Email: Alessandro Torcini* - alessandro.torcini@fi.isc.cnr.it \\ * Corresponding author
}

from Sixteenth Annual Computational Neuroscience Meeting: CNS*2007

Toronto, Canada. $7-12$ July 2007

Published: 6 July 2007

BMC Neuroscience 2007, 8(Suppl 2):PI8 doi:I0.1186/147I-2202-8-S2-PI8

(C) 2007 Torcini et al; licensee BioMed Central Ltd.

The dynamics of collective states observed in globally coupled neuronal networks is still an open problem. In particular, although it is claimed that the periodic firing state ("splay state") is stable only for excitatory coupling [1], counterexamples have been found for inhibitory coupling as well [2]. Moreover, the stability of the splay states has been analyzed only in the mean field limit $[1,3,4]$. Our aim is to investigate simultaneously, for a pulse-coupled network of leaky integrate-and fire neurons, the effect of the number $\mathrm{N}$ of neurons as well as of the pulse-width of the post-synaptic potentials. Finite- $\mathrm{N}$ networks can be studied by suitably modifying the map-like formalism $[5,6]$ usually adopted to implement numerically the model. As a result, we find that the stability of the splay state depends crucially on a parameter that is proportional to the width of the delivered pulses rescaled to the average interspike interval. More precisely, we show that the Floquet spectrum of eigenvalues is made of two components, one of which coincides with that one predicted by the mean-field analysis [1]. Depending on the value of the relevant parameter, the second component may be responsible for the occurrence of instabilities which in turn suggest the failure of the continuum limit approximation. Finally, for sufficiently small pulse-width we observe that the splay state can be stable even for inhibitory coupling.

\section{References}

I. Abbott LF, van Vreeswijk C: Asynchronous states in networks of pulse-coupled oscillators. Phys Rev E 1993, 48: I483-1490.
2. Zillmer R, Livi R, Politi A, Torcini A: Desynchronization in diluted neural networks. Phys Rev E Stat Nonlin Soft Matter Phys 2006, 74:036203.

3. van Vreeswijk C: Partial synchronization in population of pulse-coupled oscillators. Phys Rev E 1996, 54:5522-5537.

4. Mohanty PK, Politi A: A new approach to partial synchronization in globally coupled rotators. J Phys A: Math Gen 2006, 39:L4I5-L4I8.

5. Jin Z: Fast convergence of spike sequences to periodic patterns in recurrent networks. Phys Rev Lett 2002, 89:208102.

6. Bressloff PC, Coombes S: A dynamical theory of spike train transitions in networks of integrate-and-fire oscillators. SIAM J Appl Math 2000, 60:820-84I. 